ISSN: 2578-3335 (Print) 2578-3343 (Online)

\title{
Medication Literacy Tool in a Student-Run Free Clinic
}

Jessica B. Richmond, MD

Cooper University Hospital, richmond-jessica@cooperhealth.edu

Anjali Desai, MD

Cooper Medical School of Rowan University, Desai-Anjali@CooperHealth.edu

Cooper Rowan Medical Journal: https://rdw.rowan.edu/crjcsm

Would you like to be a reviewer? Please fill in this short form to express your interest.

\section{Recommended Citation}

Richmond, MD, Jessica B. and Desai, MD, Anjali (2020) "Medication Literacy Tool in a Student-Run Free Clinic," Cooper Rowan Medical Journal: Vol. 2 : Iss. 1 , Article 3.

DOI: 10.31986/issn.2578-3343_vol2iss1.3

Available at: https://rdw.rowan.edu/crjcsm/vol2/iss1/3

\section{(c) (i)}

This work is licensed under a Creative Commons Attribution 4.0 License.

This QI and Process Improvement Studies is brought to you for free and open access by the Rowan University Journals at Rowan Digital Works. It has been accepted for inclusion in Cooper Rowan Medical Journal by an authorized editor of Rowan Digital Works. For more information, please contact brush@rowan.edu. 


\section{Medication Literacy Tool in a Student-Run Free Clinic}




\title{
Medication Literacy Tool in a Student-Run Free Clinic
}

\author{
Jessica Richmond, $\mathrm{MD}^{1 *}$ \& Anjali Desai, $\mathrm{MD}^{2}$ \\ ${ }^{1}$ PGY-2-Resident Physician of Internal Medicine, Cooper University Hospital, 1 Cooper Plaza, Camden, \\ 08103, New Jersey, USA \\ ${ }^{2}$ Faculty, Department of Internal Medicine, Cooper University Hospital, 1 Cooper Plaza, Camden, 08103, \\ New Jersey, USA \\ *Corresponding author: richmond-jessica@cooperhealth.edu (Jessica Richmond, MD)
}

\begin{abstract}
Introduction: Low medication literacy can lead to prescription non-adherence and medical errors. The goal of this study was to create an easy-to-use comprehensive medication literacy assessment tool to aid in identification of patients with trouble reading and interpreting medication labels in the primary care setting. The secondary goal was to evaluate the role of primary language and education level on medication literacy and determine if patient reported difficulty with reading labels correlated with a lower medication literacy. Methods: Forty-two patients of the Cooper Rowan Clinic, a student-run free clinic, were given a demographic survey and 7-item questionnaire based on a standard prescription label. The study was limited to patients over the age of 16 who were able to read in either English or Spanish.
\end{abstract}

Results: A significant difference was noted in average scores between the English and Spanish-speaking groups. In addition, a positive correlation was found between education level and assessment scores. The assessment scores of participants who self-reported having trouble reading their label were lower but not significantly different from those who did not report difficulty reading a label. Conclusions: The assessment was easily administered in a student-run clinic and could be used for medication literacy evaluation and quality improvement in other clinics. This tool also shows that primary language and education play a role in the ability to interpret labels. Further testing could be performed to establish the validity of the assessment with longer, well-established health literacy tests.

Keywords: medication literacy, health literacy, student clinic, prescription labels

\section{INTRODUCTION}

An important element of primary care is the management of chronic conditions through medication therapy and factors such as medication nonadherence can greatly impact the health of patients. Medication 
nonadherence is known to be a multi-factorial issue influenced by access to healthcare, socioeconomic status, patient-related factors, and therapy-related factors ${ }^{1}$. The most common reasons for nonadherence demonstrated in past studies have been the cost of medications and lack of refills, however health literacy and medication literacy play a role as well ${ }^{1}$.

Health literacy is defined as the ability of an individual to access, understand, and use health-related information and services to make appropriate health decisions ${ }^{2}$. Multiple health literacy assessment tools exist in both Spanish and English and are used to assess the need for better communication between the provider and patient. For instance, the Short Assessment of Health Literacy - Spanish and English (SAHL-S\&E) is an 18-item validated health literacy word association tool used to test the comprehension and pronunciation of health-related terms by each subject. The Rapid Estimate of Adult Literacy in Medicine-Short Form (REALM-SF) is another validated tool that uses word recognition of medical terms to assess health literacy. Both tests require the administration by a trained clinician and take an average of 5 minutes to complete. Providers are encouraged to use this information to improve communication by eliminating medical jargon, repeating oral instructions, and/or creating materials with illustrations for patients who score low on these tests.

Our goal was to create a short assessment tool that could be administered without the presence of a provider using multiple choice to add in standardization and prevent variability in interpretation. Our venue, the Cooper Rowan Clinic (CRC), is a student-run free clinic located in Camden, NJ that provides care for uninsured patients. The clinic runs with an interdisciplinary team including medical and pharmacy students and features an onsite pharmacy which provides free prescription medications during patient visits, in addition to brief education on all new medications, and labels offered in either English or Spanish. However, despite these measures and the removal of cost of prescriptions as a barrier,medication nonadherence among patients at the clinic remained an ongoing issue; sparking concerns about medication literacy in the clinic patient population. Therefore, we embarked on the creation of an easy to use medication literacy assessment tool to identify patients in our clinic with difficulty reading and interpreting labels to spark further quality improvement measures.

\section{METHODS}

Study Sample: The CRC serves uninsured patients over the age of 16 who reside in the city of Camden, NJ. All patients with a scheduled appointment between the dates of March 20, 2017-April 17, 2017 were invited to participate in the survey. Patients self-reported their ability to read in English or Spanish and surveys were given in their preferred language. Patients who could not read in English or Spanish were excluded from the study. The study included a total of 42 participants. 
Survey Development: The survey was composed of two parts (Supplemental File one). Part one collected demographic information and asked the participants to rate their comfort level with reading labels, their reasons for having difficulty reading labels, self reported adverse events from incorrect medication use, and the number of medications they use daily. Part two contained a picture of a standard prescription label with 7 follow-up multiple choice questions. Amoxicillin was identified as a commonly used medication in a study by Davis et al. ${ }^{3}$ and was therefore used as the sample medication on the label for our study. The label was accompanied by a patient scenario to assist in providing context: "Jane Smith was prescribed an antibiotic medication called Amoxicillin on 6/1/2012 for a bacterial infection." The 7item assessment was a multiple choice section developed to ask specific questions about the label. Scores were given based on the total number of correct items on the assessment (score 0-7). The survey was developed using the four domains of literacy required to read and understand how to use labels: prose, document, numeracy, and problem-solving ${ }^{4}$. Prose literacy is the ability to understand and apply information from text (e.g. newspapers, brochures, editorials). Document literacy is the ability to locate and use information contained in various formats (e.g. maps, tables, charts). Numeracy is the ability to manage and calculate numbers. Problem-solving requires the ability to think and act in situations where there is no routine solution available.

The original question set was vetted and altered based on participant feedback of language and format. The average time to complete the 7-item questionnaire was 5-10 minutes and completed independently by each participant. (Supplemental File one. Parts One and Two of Survey located in the Appendix.)

Statistical analysis: Chi-square tests were used for the following comparisons of assessment scores: English vs. Spanish-speaking participants, participants answering "yes" to having trouble reading a label vs. participants answering “no", and stratification based on education level. Spearman's rank-order correlation was used to look at the relationship between education level and average assessment scores due to the inconsistency in years between different education levels. To calculate Spearman, a value of 0-3 was given for each education level: $0=$ no schooling, $1=$ elementary school, $2=$ high school, and $3=$ college. Similar to Pearson's coefficient, Spearman's is interpreted on a scale value of -1(negative relationship) to +1 (positive relationship).

\section{RESULTS}

Groups were separated according to the type of survey used (English vs. Spanish) for the purpose of data analysis and comparison.

Demographics: The mean age across all groups in the study was 47.1. The study was made up of 57.9\% female participants and $42.1 \%$ male participants. The majority of participants identified as 
Hispanic/Latino (69.1\%) with African-American/black being the second highest (19\%). The most common primary languages spoken were Spanish (54.8\%) and English (38.1\%), with Other at 7.1\%. The average number of medications taken daily by participants was 2.7 .

Additional Information: Only 6\% of participants reported taking their medications incorrectly in the past, and $0 \%$ reported having a known adverse event related to medication nonadherence. Participants were asked to self-identify why they felt they had trouble reading medication labels. Fourteen participants self-identified as having trouble reading their medication labels and reasons for the difficulty are illustrated inFigure 1 .

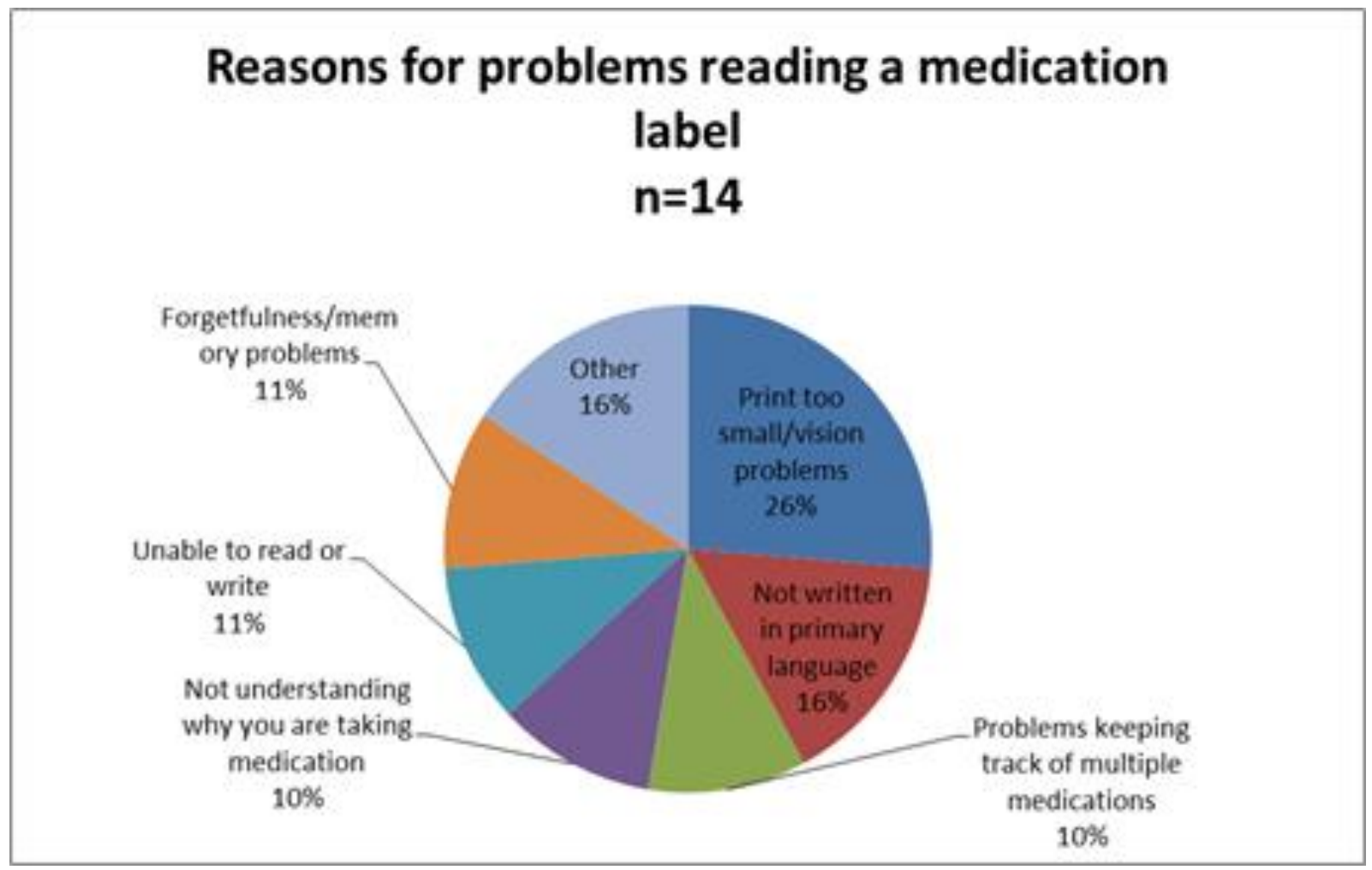

Figure 1 Reasons for problems reading a medication

Education level distribution: The highest level of education for the Spanish-speaking group was as follows: $19 \%$ no schooling, $42.9 \%$ elementary school level or equivalent, and $38.1 \%$ completed some high school or received a high school diploma. In comparison, the highest level of education for the English-speaking group was as follows: $60 \%$ completed some high school or received a high school diploma, and $40 \%$ completed college level courses.

Assessment score comparison: The combined average score on the 7-item assessment for all participants in this study was a 4.7 out of 7. The average score on the 7-item assessment between each group was 6.4 for English-speaking $(n=17)$ and 3.5 for Spanish-speaking $(n=25)$ with a $p$ value of <.0001. When combining the data for both groups (Figure 2 ), there was a positive relationship between education level 
and assessment scores with Spearman's correlation of +1 ( $p$ value $=0.0003$ ); however, there was no significant difference between education level and assessment scores when separated by ethnic group. The average score breakdown by education level was 1.5 for those with no schooling, 4.11 for elementary school level, 5.06 for high school level, and 6.29 for college level. Participants who reported having trouble reading their label had assessment scores that were lower but not significantly different from those who reported that they did not have trouble reading a label.

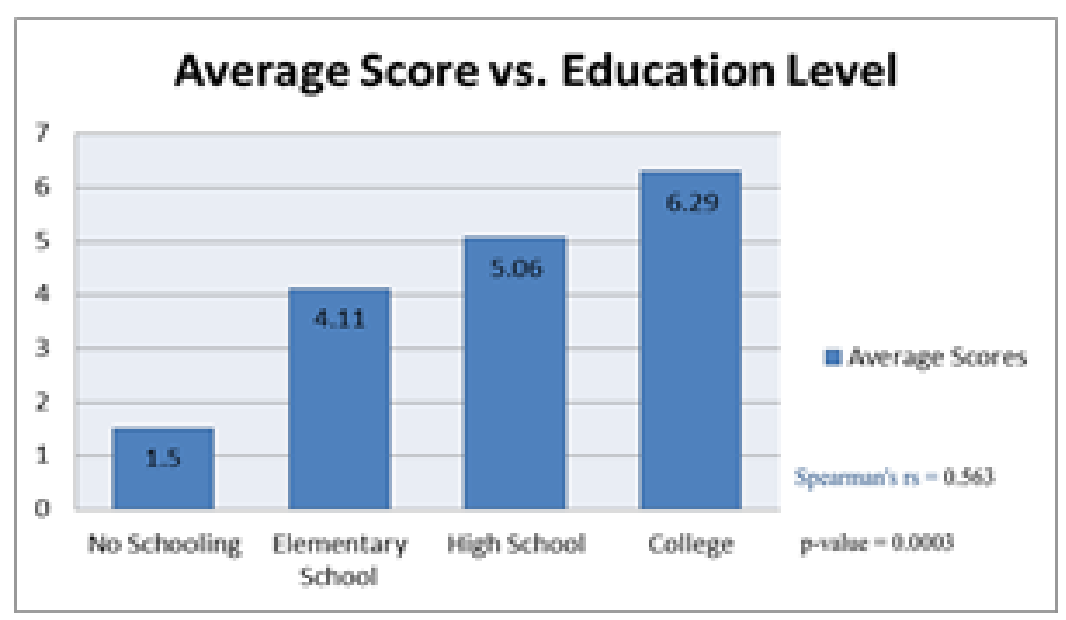

Figure 2 Correlation between average score and education level. Lowest possible score being a zero and highest possible score being a 7 .

The components of the assessment were also examined to see which questions were most frequently missed. Incorrect questions most often involved problem-solving skills and utilized numbers. For example, "how many total milligrams (dosage) should Jane take each day", was missed by $53.7 \%$ of participants. The majority of participants chose $500 \mathrm{mg}$ instead of $1500 \mathrm{mg}$, indicating a misunderstanding of how to interpret and analyze dosage on a bottle. The second most frequently missed question asked the following: “A year later on 6/27/2013, Jane gets sick again and notices she has a few pills of Amoxicillin left over from before. What should Jane do with the medication?" Thirty-two percent of participants answered this question incorrectly. This could indicate that participants had some difficulty realizing that Jane did not take her medication correctly the first time; as well as not noting the expiration date on the bottle.

\section{DISCUSSION}

As of recent, a newer term coined as "medication literacy" was born out of the concept of health literacy and defined as the ability to obtain, evaluate, calculate, and comprehend basic information about pharmacotherapy to make appropriate medication-related decisions; regardless of the mode of content delivery (e.g. written, oral, visual images and symbols $)^{5}$. Both types of literacy are vital to navigating the 
healthcare system and improving health status, but understanding instructions on a medication label presents a particular challenge for those who have low health literacy 5 .

Davis et al. set out to identify patients with trouble reading prescription labels by using an interview style assessment ${ }^{3}$. The population was English speaking only and six primary care providers with one pharmacist interviewed a total of 356 patients using 5 container labels. The providers asked each patient to demonstrate how they would use the medication on the label. Findings from this study showed that low health literacy and medication literacy was significantly associated with misunderstandings of how to use medication, and there was a relationship between lower education level and low medication literacy. However, they excluded Spanish-speakers in their study. Following this study, in 2012, Sauceda came up with a medlitRx assessment tool that also used interview style questions with multiple clinical scenarios and labels ${ }^{6}$. Research assistants read test items to patients and wrote down their response focusing on questions that targeted multiple areas of literacy: document literacy, numeracy, and general literacy. This study also included both English and Spanish speakers.

Both Davis and Saucedo were amongst the first to try to directly assess patients' understanding of reading medication labels, but both required face-to-face interaction with a provider to administer their tool. In addition, Davis demonstrated variability from one provider to the next when interpreting answers to open-ended questions ${ }^{3}$. Our study set out to address the limitations of these previous studies by creating an easy to use but comprehensive medication literacy assessment tool that could be easily administered in a clinical setting. Our assessment tool does not require face-to-face interaction and there is less room for provider bias with multiple choice vs. open-ended questions that were used in other studies. In addition, we chose labels written in either English or Spanish to reflect our population and to eliminate language barriers. Data collection established a baseline and the ability to analyze the parameters that have an effect on how our patients read medication labels. One particular finding was the impact of education level and primary language spoken. There was a significant difference in assessment scores and education level between Spanish and English-speaking populations. As demonstrated from previous studies, our study also showed that higher education was correlated with higher assessment scores; which is an indication that literacy plays a significant role in how well patients can read and interpret their medication label. When educating patients on how to use their medication, the focus is generally on explaining the diagnosis, side effects, and how many capsules to take daily. However, this study demonstrates a need to better educate patients on how to read and interpret the different components of a label. For instance, the most frequently missed questions were those involving numeracy; particularly understanding that one pill equaled the dosage amount written on the label. This is particularly important when patients transition across multiple health institutions and are asked how much of their medications they are taking. 
Misinterpretation of this can lead to undertreatment or potential adverse outcomes of over or underdosing medications; especially in cases where providers are making dosage adjustments.

We were not able to determine the risk of having an adverse event in relation to assessment scores as none were reported by participants in this study. It is also possible that patients would be unaware of having an adverse event if they did not correlate those symptoms with taking the medication incorrectly. For instance, a patient taking too many antihypertensive pills may experience feeling light-headed from hypotension but fail to realize this is due to their medication if they have other possible causes for their symptoms. Therefore, it may benefit providers to investigate how patients are taking their medications if they report side effects or if there is a lack of improvement in their health status.

Our study was limited by the small sample size. Only 42 participants completed the 7 item assessment. Further studies could include a larger sample size to further assess the relationship between education level vs. assessment scores for each ethnicity group. This will account for the differences in length and quality of education for those who are not native-born.

\section{CONCLUSION}

This 7-item assessment was easily administered in a student-run clinic. Similar to previous studies, our tool demonstrated that education plays a role in the ability to read medication labels. In addition, having labels written in one's preferred language is not enough to address how well patients can understand their label; particularly since the dosage amount written on the bottle and expiration date as those were the most misinterpreted components of the label. Future studies should be conducted to address the limitations of this study and to validate the 7-item assessment with the use of well-established health literacy tests.

\section{Practice Implications}

This more user friendly survey could be used in other settings to identify patients at-risk for medication non-adherence or medical errors. This tool also offers a way for providers to make a quick assessment of patients' medication literacy when there is lack of clinical improvement or adverse side effects from medical therapy. Data from the use of the surveys could spark quality improvement opportunities in other clinics and alter how providers counsel their patients on reading medication labels.

\section{REFERENCES}

1. Bailey Genee R., Barner Jamie C., Weems James K., et al. Assessing Barriers to Medication Adherence in Underserved Patients With Diabetes in Texas. The Diabetes Educator. 2012;38(2):271-279. 
2. Nielsen-Bohlman L, Panzer A M, Kindig D A. Health Literacy: A Prescription to End Confusion. Washington (DC: National Academies Press; .

3. Davis Terry C., Wolf Michael S., Bass Pat F., et al. Literacy and Misunderstanding Prescription Drug Labels. Annals of Internal Medicine. 2006;145(12):887-887.

4. Weiss B. D.. Quick Assessment of Literacy in Primary Care: The Newest Vital Sign. The Annals of Family Medicine. 2005;3(6):514-522.

5. Pouliot Annie, Vaillancourt Régis, Stacey Danielle, Suter Philippe. Defining and identifying concepts of medication literacy: An international perspective. Research in social \& administrative pharmacy : RSAP. 2018;14(9):271-279.

6. Sauceda John A., Loya Amanda M., Sias Jeri J., Taylor Thomas, Wiebe John S., Rivera José O.. Medication literacy in Spanish and English: Psychometric evaluation of a new assessment tool. Journal of the American Pharmacists Association. 2012;52(6):e231-e240. 


\section{APPENDIX}

Supplemental File one: Parts One and Two of Survey.

Demographic Characteristics

1 Gender

O Male

O Female
Other

2 Age

O 18.24

O 25.34

O 35.44

O $45-54$

O 55.64

65.74

075 or older

3 Race/Ethnicity

O White/Caucasian

OHispanic or Latino

O Black or African American

Native American or American Indian

O Asian / Pacific islander

O Other

4 Highest level of Education

O No schooling completed

O Nursery school to 8th grade

O Some high school, no diploma

High school graduate, diploma or the equivalent

(for example: GED)

O Some college credit, no degree

Orade/technical/vocational training

O Associate degree

O Bachelor's degree

O Master's degree

O Professional degree

O Doctorate degree 
5 What is the primary language you speak at home?

6 What is the primary language you read and write?

7 Rate your comfort level with reading medication labels in English.

9 Rate your comfort level with being able to understand instructions on your medication labels in English.

8 Rate your comfort level with reading medication labels in Spanish (if applicable).

10 Rate your comfort level with being able to understand instructions on your medication labels in Spanish (if applicable)

11 Have you ever had problems reading or understanding your medication labels?

12 If yes to the above question, please select the reason(s). Select all that apply.

13 Other (please specify)

Does someone help you read your medication label?

14 Have you ever taken your medications incorrectly?

15 If yes to the above question, please state whether you had any negative outcomes due to incorrect administration of medication.

(Example: overdose, unpleasant side effects, lack of relief from symptoms, etc.)

Specify the negative outcome

16 How many medications do you take daily?

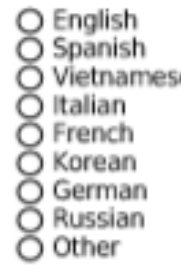

Onglish

OSpanish

Vietnamese

Otalian

O French

O Korean

Oerman

Ỏ Russian

Other

O Very uncomfortable

OSomewhat uncomfortable

Unsure

O Somewhat comfortable

O very comfortable

V Very uncomfortable $O$ Somewhat uncomfortable $\mathrm{O}$ Unsure

Somewhat comfortable

O very comfortable

Very uncomfortable

OSomewhat uncomfortable

O Unsure

Somewhat comfortable

V very comfortable

O Very uncomfortable $O$ Somewhat uncomfortable $\mathrm{O}$ Unsure O Somewhat comfortable $\bigcirc$ very comfortable

OYes ONo

$\square$ Print is too small / Vision problems

Not written in primary language

Problems keeping track of muitiple medications $\square$ Not understanding why you are taking the medication

Unable to read or write

Forgetfulness/memory problems

other

Yes
No

OYes O No

O Yes O No 


\section{This part of the survey will ask you a series of 7 questions to test your ability to interpret a} sample medication label.

Read the clinical scenario first and use the medication label to help answer the questions below.

Clinical Scenario:

Jane Smith was prescribed an antibiotic medication called Amoxicillin on 6/1/2012 for a bacterial infection. She was given the following medication label and asked to follow the written instructions.

\begin{tabular}{|c|c|}
\hline 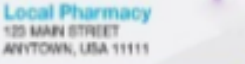 & $\begin{array}{l}\text { Che. Doves } \\
\text { (3000) 555-8555 }\end{array}$ \\
\hline so 0060023-08291 & 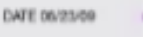 \\
\hline \multicolumn{2}{|l|}{$\begin{array}{l}\text { JANE SMITH } \\
\text { as แaผ }\end{array}$} \\
\hline \multicolumn{2}{|c|}{ 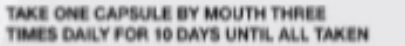 } \\
\hline 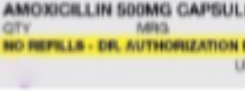 & oumas \\
\hline
\end{tabular}

1 How many total capsules should jane take each day?

2 How is the medication administered?

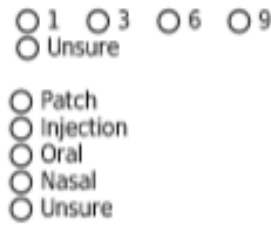

3 How many total milligrams (dosage) should jane take each day?

$$
\begin{array}{ll}
\text { O } 500 \mathrm{mg} & \text { O } 1500 \mathrm{mg} \\
\text { O } 1800 \mathrm{mg} & \text { O } 2000 \mathrm{mg} \\
\text { O Unsure } &
\end{array}
$$

4 How often should jane take the medication?

O As needed O Every day until she needs a

refill $O$ Every day until she is finished

The entire bottle in one day

O Unsure

5 If Jane started the medication on $6 / 1 / 2012$, which date will she take her last dose (assuming she did not miss a dose)?

6 Jane is starting to feel better on 6/5/2012 but she still has more capsules left. is it ok for her to stop her medication?

7 A year later on 06/27/2013, Jane gets sick again and notices she has a few pills of Amoxicillin leftover from before. What should jane do with the medication?

O. 6/10/2012 $\mathrm{O} 6 / 2712 / 2012$

O Unsure

OYes O No OUnsure

O Take by mouth until medicine is finished Double the amount of capsules she takes each day $\mathrm{O}$ Reduce the amount of capsules she takes each day by half

O Discard or stop taking the medication

O Unsure 